\title{
Calculation of the Hubble Constant and the Minimum Mass Using the Dirac's Hypothesis on the Ratio of the Electrostatic Force to the Gravitational Force (Preprint)
}

Paul Talbot

DOI : $\underline{\text { https://osf.io/yuhw7/ }}$

St-Hubert, Qc, Canada

ORCID: 0000-0002-9919-9034

\begin{abstract}
This publication suggests that some physical values could be calculated using the Dirac's hypothesis on the observed ratio of the electrostatic force to the gravitational force. The calculated value of the Hubble constant is $H \approx 72.013 \mathrm{~km} \mathrm{~s}^{-1} \mathrm{Mpc}^{-1}$ and that of the minimum mass $M_{\min } \approx 1.7206 \times 10^{-68} \mathrm{~kg}$.
\end{abstract}

\section{Keywords}

Cosmology, Hubble constant, minimum mass, graviton, dimensionless constants.

\section{Introduction}

Currently, the local value of the Hubble constant is determined by observation. The observed values are more or less precise, and oscillate between $70 \mathrm{~km} \mathrm{~s}^{-1} \mathrm{Mpc}^{-1}$ and $74 \mathrm{~km} \mathrm{~s}^{-1} \mathrm{Mpc}^{-1}$, depending on the techniques used [1] [2] [3] [4] [5] [6].

We know that some physical quantities are limited by minimum values. For example, the quantum of action $h$ is the minimum value of action. The elementary charge $e$ can also be regarded as the minimum charge. Quarks are associated with a fractional charge, but they are confined inside hadrons, which do have an integer charge [7].

\section{Hypothesis About Minimum Values}

The working hypothesis is that other physical quantities would also be limited by minimum values (subscript min). Using algebraic equations and the observed ratio of the electrostatic force to the gravitational force, some minimum values could be calculated precisely.

The Hubble constant $H$ (of dimension $\mathrm{T}^{-1}$ ) would correspond to the minimum frequency:

$$
f_{\min }=H \text {. }
$$

Since $E=h f$, the minimum energy would be

$$
E_{\min }=h f_{\min }=h H .
$$

Since $M=E / c^{2}$, the minimum mass would be

$$
M_{\min }=E_{\min } / c^{2}=h H / c^{2} .
$$

To calculate $H$, another, but more precise value must be involved. Since May 2019, the value of the elementary charge is fixed by definition at $e=1.602176634 \times 10^{-19} \mathrm{C}$. 


\section{The Ratio of the Electrostatic Force to the Gravitational Force}

The ratio, say $\beta$, of the electrostatic force to the gravitational force between an electron and a proton is

$$
\beta=e^{2} / 4 \pi \varepsilon_{0} G m_{\mathrm{e}} m_{\mathrm{p}} \approx 2.26866 \times 10^{39}
$$

$\varepsilon_{0}$ is the vacuum permittivity.

$G$ is the gravitational constant .

$m_{\mathrm{e}}$ is the mass of the electron.

$m_{\mathrm{p}}$ is the mass of the proton.

In the last century, Paul Dirac popularized this $\beta$ ratio in a cosmological context [8] [9] [10]. The value of the $\beta$ ratio depends on the masses of the interacting particles. Here, I stick to the natural choice made by Dirac (the electron and the proton). In this context, these two particles are regarded as two sides of the same coin: the electron-proton couple.

The gravitational force is tiny compared to other forces, so this $\beta$ ratio is sometimes called a dimensionless "Large Number". It still gets attention nowadays [11] [12] [13] [14].

\section{Calculation of $\boldsymbol{H}$ Using the Minimum Energy}

Gravitational and electrostatic forces are conservative, which implies that the $\beta$ ratio applies to both forces and energies. An energy $E_{\mathrm{ep}}$ can thus be associated with the electron-proton couple:

$$
E_{\mathrm{ep}}=\beta E_{\min }=\beta h H,
$$

that could be calculated precisely using the assumed relation

$$
E_{\mathrm{ep}}^{2}=m_{\mathrm{e}} m_{\mathrm{p}} c^{4}
$$

where $c$ is the speed of light in vacuum. Using the 2018 CODATA recommended values, we get

$$
E_{\mathrm{ep}}=\sqrt{m_{\mathrm{e}} m_{\mathrm{p}}} c^{2} \approx 3.5082 \times 10^{-12} \mathrm{~J}, \text { or } 22.346 \mathrm{MeV}
$$

Since $E_{\mathrm{ep}}=\beta h H$, we deduce that

$$
H=E_{\mathrm{ep}} / \beta h \approx 2.3338 \times 10^{-18} \mathrm{~s}^{-1} \text {, or } 72.013 \mathrm{~km} \mathrm{~s}^{-1} \mathrm{Mpc}^{-1},
$$

halfway between low and high observed values.

This means that a frequency $f_{\text {ep }}$ can be associated with the electron-proton couple:

$$
f_{\mathrm{ep}}=\beta H=E_{\mathrm{ep}} / h \approx 5.2945 \times 10^{21} \mathrm{~s}^{-1} .
$$

Expanding equation (8), we note that physical constants would be interlinked:

$$
H=4 \pi \varepsilon_{0} G\left(m_{\mathrm{e}} m_{\mathrm{p}}\right)^{3 / 2} c^{2} / h e^{2} .
$$

The value of the minimum energy would be

$$
E_{\min }=h H \approx 1.5464 \times 10^{-51} \mathrm{~J},
$$

and that of the minimum mass

$$
M_{\min }=h H / c^{2} \approx 1.7206 \times 10^{-68} \mathrm{~kg} .
$$

The very definition of the $\beta$ ratio suggests that this tiny mass might be that of the graviton. 


\section{Previous Calculations of Minimum Values}

Remarkably, the value of $M_{\min }$ coincides with the Wesson's mass ( $m_{\mathrm{E}} \approx 2 \times 10^{-68} \mathrm{~kg}$ ), i.e., the quantum of mass calculated by Pr. Wesson [15] in 2003 using the constants $c, h$ and $\Lambda$ (the cosmological constant). According to Wesson, $m_{\mathrm{E}}=(h / c)(\Lambda / 3)^{1 / 2}$, compared to $M_{\min }=H h / c^{2}$ under the present hypothesis. We deduce that

$$
\Lambda=3 H^{2} / c^{2} \approx 1.818 \times 10^{-52} \mathrm{~m}^{-2},
$$

according to Wesson's $\Lambda$ hypothesis. In the conformal cosmological model $(\Lambda \mathrm{CDM})$, this value is multiplied by a factor $\Omega_{\Lambda} \approx 0.7$ [16] [17].

Using dimensional analysis, Pr. Dimitar Valev [18] [19] [20] [21] formulated an equation for a minute mass that could be that of the graviton: $m_{H} \approx \hbar H / c^{2}$. This tiny mass is of the same order of magnitude as the minimum mass $M_{\min }$.

Likewise, using dimensional analysis, Pr. Ali Muhammad Rushdi, and Dr. Mostafa Ali Rushdi [22] deduced a dimensionless product $\left(\pi_{a 4}=h H / m_{2} c^{2}\right)$ where $m_{2}$ would equal to $\mathrm{M}_{\min }$ when $\pi_{a 4}=1$.

In 2021, Mr. Ramón Garza Wilmot posted a research on ResearchGate: «Exact Value of the Hubble Constant - The Most Precise Value of the Hubble Constant Deduced from Other Constants of Physics» [23]. Although the reasoning differs and implies a fictitious particle called «mason», the calculations and the resulting values of $H$ and $M_{\min }$ agree. Mr. Wilmot's work was posted on September 8, 2021, five days before mine on Amazon [24] [25]. The anteriority of the calculation of $H$ and $M_{\min }$ thus belongs to Mr. Wilmot.

Among related research, some calculated results come close to the suggested value of $\mathrm{H}$ :

This work (72.013 $\left.\mathrm{km} \mathrm{s}^{-1} \mathrm{Mpc}^{-1}\right)$,

Dr. Kritov's [26] (71.995 $\left.\mathrm{km} \mathrm{s}^{-1} \mathrm{Mpc}^{-1}\right)$,

Mr. Mercier's [27]

$\left(72.095 \mathrm{~km} \mathrm{~s}^{-1} \mathrm{Mpc}^{-1}\right)$,

Mr. Wolf's [28]

(71.994 $\left.\mathrm{km} \mathrm{s}^{-1} \mathrm{Mpc}^{-1}\right)$. 


\section{Chart of the Assumed Relations Between Physical Values}

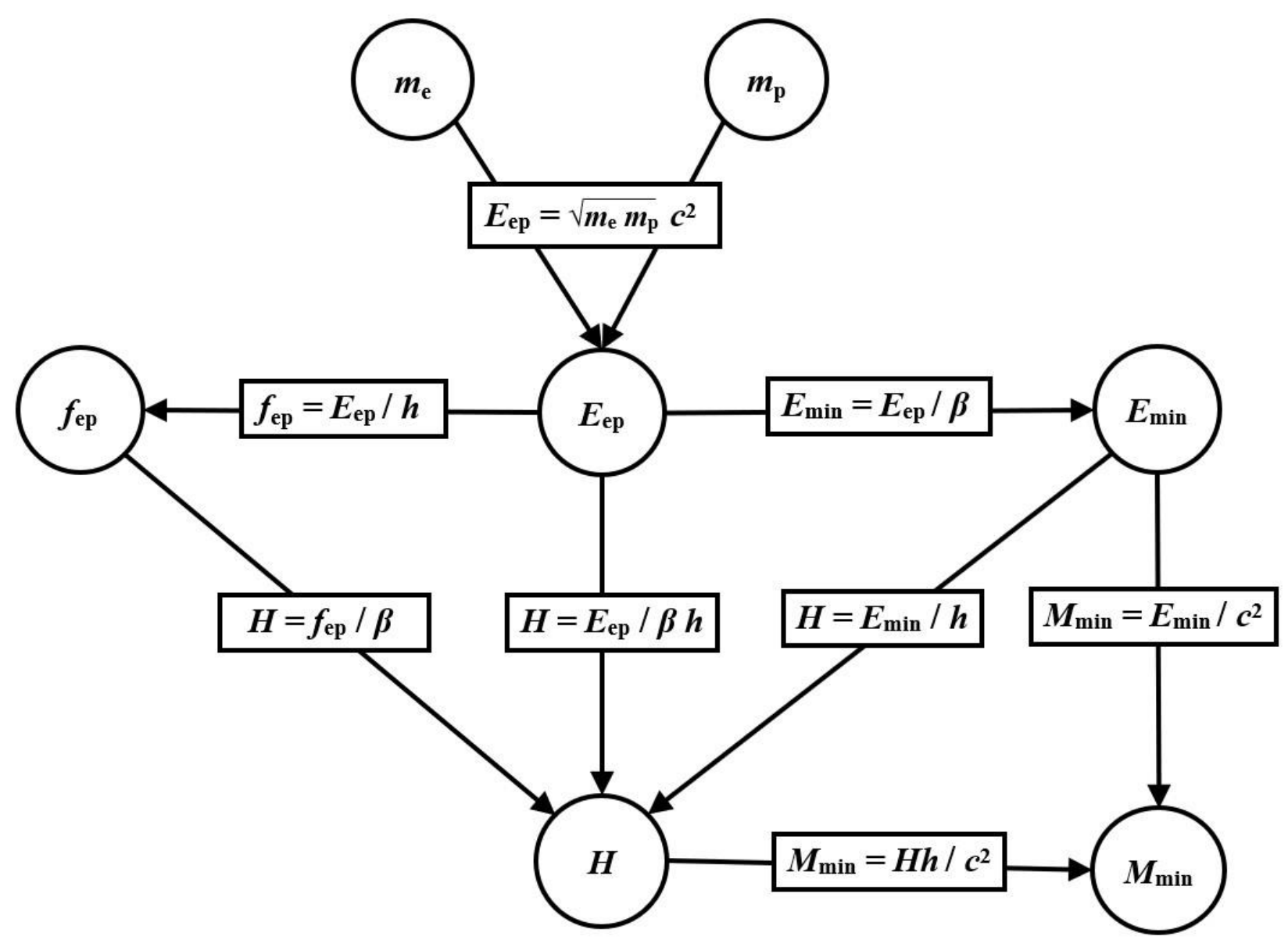

Figure 1: Assumed relations between physical values

Physical values are represented by circles, and the relationships between them by arrows, each with a boxed formula. Each arrow indicates the direction of the relationship expressed by the formula linking two quantities. This direction is arbitrary and can be reversed by changing the formula. For example, the relation $\left(M_{\min }=H h / c^{2}\right)$ can be written $\left(H=M_{\min } c^{2} / h\right)$.

\section{Conclusion}

Using the observed ratio of the electrostatic force to the gravitational force, the assumed relation (6) allows precisely calculating the Hubble constant and the minimum mass. These values agree with observations and previous works. It is unlikely that an arbitrary of $E_{\text {ep }}$ could lead to such consistent results. More precise observed values of $H$ could confirm or refute this hypothesis. 


\section{References}

[1] A. Riess, Y. Wenlong, M. Lucas, M. Macri, D. Scolnic, D. Brout, S. Casertano, D. O. Jones, Y. Murakami, L. Breuval, T. G. Brink, A. V. Filippenko, S. Hoffmann, S. W. Jha, W. D'arcy Kenworthy, J. Mackenty, B. E. Stahl et W. Zheng, "A Comprehensive Measurement of the Local Value of the Hubble Constant with $1 \mathrm{~km} \mathrm{~s}-1 \mathrm{Mpc}-1$ Uncertainty from the Hubble Space Telescope and the SHOES Team, » arXiv:2112.04510v2 [astroph.CO] 10 Jan 2022, p. 67, 2022.

[2] W. Freedman, «Measurements of the Hubble Constant: Tensions in Perspective,» The Astrophysical Journal, vol. 919:16, p. 22, 2021.

[3] N. Khetan, L. Izzo, M. Branchesi, R. Wojtak, M. Cantiello, C. Murugeshan, A. Agnello, E. Cappellaro, M. Della Valle, C. Gall, J. Hjorth, S. Benetti, E. Brocato, J. Burke, D. Hiramatsu, D. A. Howell, L. Tomasella et S. Valenti, "A new measurement of the Hubble constant using Type la supernovae calibrated with surface brightness fluctuations," Astronomy \& Astrophysics, vol. 647, n%1A72, p. 20, 2021.

[4] K. Liao, A. Shafieloo, R. E. Keeley et E. V. Linder, "A Model-independent Determination of the Hubble Constant from Lensed Quasarsand Supernovae Using Gaussian Process Regression, " The Astrophysical Journal Letters, vol. 886, n%1L23, p. 7, 2020.

[5] D. W. Pesce, J. A. Braatz, M. J. Reid, A. G. Riess, D. Scolnic, J. Condon, F. Gao, C. Henkel, C. M. V. Impellizzeri, C. Y. Kuo et K. Y. Lo, «The Megamaser Cosmology Project. XIII. Combined Hubble Constant Constraints," The Astrophysical Journal Letters, vol. 891, n%1L1, p. 9, 2020.

[6] T. Yang, S. Birrer et B. Hu, «The first simultaneous measurement of Hubble constant and postNewtonian parameter from Time-Delay Strong Lensing, " astro-ph.CO, vol. MNRASS Preprint, p. 6, 2020.

[7] M. L. Perl, E. R. Lee et D. Loomba, «A Brief Review of the Search for Isolatable Fractional Charge Elementary Particles, » Modern Physics Letters, vol. 19.35, p. 16, 2004.

[8] P. A. Dirac, "A new basis for cosmology," Proceedings of the Royal Society of London, vol. 165, pp. 199208, 1938.

[9] P. A. Dirac, «Cosmological Models and the Large Numbers Hypothesis,» Proceedings of the Royal Society of London, vol. 338, pp. 439-446, 1974.

[10] P. Dirac, «The Large Numbers hypothesis and the Einstein theory of gravitation," Procedings of the Royal Society, n%1365, pp. 19-30, 1979.

[11] Saibal Ray, Utpal Mukhopadhyay, Soham Ray et Arjak Bhattacharjee, «Dirac's large number hypothesis: A journey from concept to implication, " International Journal of Modern Physics $D$, vol. $28, \mathrm{n}^{\circ} \% 108$, 2019.

[12] A. Cetto, L. de la Peña et E. Santos, «Dirac's large-number hypothesis revised,» Astronomy and Astrophysics, vol. 164, pp. 1-5, 1986.

[13] M. S. Berman , "Large number hypothesis, International Journal of Theoretical Physics, vol. 31, pp. 1447-1450, 1992. 
[14] Y. K. LauA; S. J. Prokhovnik; "The Large Numbers Hypothesis and a Relativistic Theory of Gravitation," Australian Journal of Physics, vol. 39, pp. 339-346, 1986.

[15] P. S. Wesson, «Is mass quantized,» University of Waterloo, Waterloo, CA, 2003.

[16] E. Lusso, E. Piedipalumbo, G. Risaliti, M. Paolillo, S. Bisogni, E. Nardini et L. Amati, «Tension with the flat $\Lambda C D M$ model from a high-redshift Hubble diagram of supernovae, quasars, and gamma-ray bursts,》 Astronomy \& Astrophysics, vol. 628, n%1L4, p. 5, 2019.

[17] M. Shuntov, «Unveiling the Concordance Model of Cosmology, A Aix Marseille Universit'e, Marseille, 2018.

[18] D. Valev, «Three Fundamental Masses Derived by Dimensional Analysis,» American Journal of Space Science, pp. 145-149, 2013.

[19] D. Valev, "Evidence of Dirac Large Numbers Hypothesis,» PROCEEDINGS OF THE ROMANIAN ACADEMY, vol. 20, n%14, pp. 361-368, 2019.

[20] D. Valev, «Neutino and Gravito Mass Estimations by a Phenomenological Approach,» Aerospace Research in Bulgaria, vol. 22, 2008.

[21] D. Valev, «Estimations of Neutrino and Graviton Masses by a Phenomenological Mass Relation for Stable Particles,» Physics International, pp. 82-88, 2015.

[22] M. A. Rushdi et A. M. Rushdi, «On the Fundamental Masses Derivable by Dimensional Analysis,» Journal of King Abdulaziz University : Engineering Sciences, vol. 27, pp. 35-42, 2016.

[23] R. G. Wilmot , «Exact Value of the Hubble Constant - The Most Precise Value of the Hubble Constant Deduced from Other Constants of Physics, ") ResearchGate, p. 28, 2021.

[24] P. Talbot, The Cosmopheric Principle, St-Hubert, Qc, Canada: Paul Talbot (Amazon), 2021.

[25] P. Talbot, Le principe cosmosphérique, St-Hubert, Qc, Canada: Paul Talbot (Amazon), 2021.

[26] A. Kritov, «Explicit Values for Gravitational and Hubble Constants from Cosmological Entropy Bound and Alpha-Quantization of Particle Masses,» Progress in Physics, vol. 17, pp. 158-163, 2021.

[27] C. Mercier, «Calculation of the Universal Gravitational Constant, of the Hubble Constant, and of the Average CMB Temperature,» Journal of Modern Physics, vol. 10, pp. 641-662, 2019.

[28] C. G. Wolf, «Hubble constant $\mathrm{HO}$ is derived from Newtonian gravitational constant G, speed of light in vacuum c, electron mass me, classical electron radius re squared and fine structure constant $\alpha, \nu$ ResearchGate, p. 7, 2022.

[29] D. Valev, «Neutino and Gravito Mass Estimations by a Phenomenological Approach,» Aerospace Research in Bulgaria, vol. 22, 2008. 